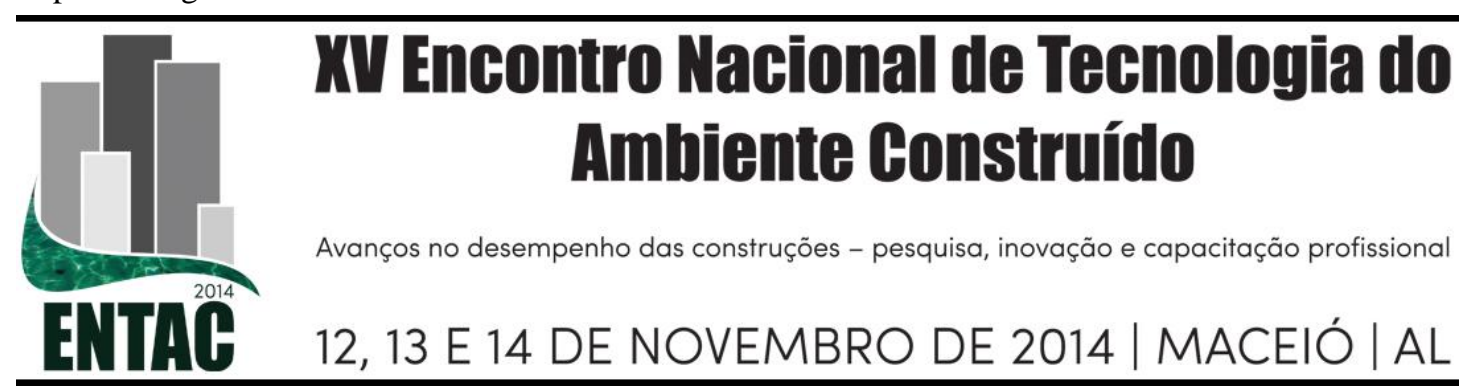

\title{
MUDANÇA DE PARADIGMA DA DEFESA CIVIL EMBASADO NO CONCEITO DE RESILIÊNCIA URBANA
}

FERREIRA, Karolyne (1); ABIKO, Alex (2)

(1) Escola Politécnica da Universidade de São Paulo, e-mail: karolyne.ferreira@ usp.br (2) Escola Politécnica da Universidade de São Paulo, e-mail: alex.abiko@poli.usp.br

\begin{abstract}
RESUMO
Os desastres naturais expõem vulnerabilidades das cidades tanto em questões de prevenção quanto de respostas aos mesmos. Considera-se um desastre natural quando um fenômeno natural atinge estruturas socioeconômicas, ou seja, quando trás danos materiais e à vida humana. Os desastres naturais mais recorrentes no Brasil são: estiagem e seca, inundações e alagamentos.

O conceito de resiliência urbana tem embasado discussões relativas à redução dos riscos de desastre. Em um ambiente urbano, resiliência é entendida em termos humanos e de infraestrutura como preparação, adaptação, resposta e recuperação de uma cidade frente a um choque externo de forma eficiente para se restabelecer um equilíbrio.

Este conceito foi adotado pela Defesa Civil. No Brasil, esta instituição é responsável pelas ações de prevenção, preparação, mitigação, resposta e recuperação. Historicamente, as ações da Defesa Civil eram mais evidentes na fase de resposta, mas a partir do Programa 2040 - Gestão do Risco e Resposta a Desastres, que faz parte dos programas e ações da Defesa Civil nacional, observou-se uma mudança de paradigma, voltada principalmente para ações de preparação e prevenção.

Este trabalho tem por objetivo analisar essa mudança de paradigma, de forma a levantar pontos importantes na discussão sobre maneiras de pensar o espaço urbano frente aos desastres naturais.

O Programa 2040, no ano de 2012 investiu em ações de prevenção mais de R 360 milhões e no ano de 2013, foram mais de R $\$ 740$ milhões investidos.

Análises preliminares mostram que ao adotar o conceito de resiliência urbana às suas ações a Defesa Civil passa a priorizar a gestão do risco. Os investimentos foram em obras de drenagem, contenção de encostas, controle de cheias e monitoramento e alerta.
\end{abstract}

Palavras-chave: Resiliência Urbana, Gestão do Risco, Defesa Civil.

\begin{abstract}
Natural disasters expose vulnerabilities of cities both in matters of prevention and response. A natural disaster is a natural phenomenon that affects negatively both infrastructure systems and population. The most recurrent natural hazards in Brazil are: drought and flooding.

The concept of urban resilience has based discussions on disaster risk reduction. In an urban environment resilience is understood in terms of human and infrastructure in preparation, adaptation, response and recovery of a city from an external shock efficiently to restore the balance.
\end{abstract}

This concept was adopted by the Civil Defense. In Brazil this institution is responsible for prevention, preparedness, mitigation, response and recovery from disasters.

Historically the Civil Defense were more evident in the response phase, but from the Program 2040 - Risk Management and Disaster Response, which is part of the programs and actions of the national Civil Defense a paradigm shift was observed, mainly focused on preparedness and prevention actions. 
This study aims to analyze this paradigm shift to raise important points in the discussion of ways of thinking about urban space regarding natural disasters.

The Program 2040 in 2012 invested in prevention actions over $R \$ 360$ million, in the year 2013 were more than $R \$ 740$ million invested.

Preliminary analyzes has shown that by adopting the concept of urban resilience to their actions Civil Defense shall prioritize risk management. The investments were in urban drainage, slope stabilization, flood control and monitoring and alerting.

Keywords: Urban Resilience, Disaster Risk Management, Civil Defense.

\section{INTRODUÇÃO}

No Brasil os desastres naturais mais recorrentes são estiagem e seca, inundações e alagamentos. Entre 1991 - 2010 a Região Nordeste, Região Sul e Região Sudeste foram as mais atingidas por desastres (CEPED/UFSC, 2012).

Inúmeros desastres naturais são reportados na mídia todos os anos, a maioria deles com vítimas. Eventos extremos que causaram tragédias a exemplo das enchentes em Pernambuco e Alagoas, em 2010 (FIALHO; MOLION, 2011); e os deslizamentos na Região Serrana do Rio de Janeiro em 2011, mostraram a Defesa Civil presente no momento da resposta, de socorro às vitimas e na contabilização dos danos humanos e materiais.

O conceito de resiliência urbana nas discussões de planejamento urbano tem forte presença no que se refere à prevenção, preparação, ação, resposta e recuperação de desastres naturais.

Entende-se por desastre natural como a ocorrência de um fenômeno natural (chuvas, terremotos, furacão, etc.) atingir e causar graves danos a estruturas socioeconômicas. (ALCÁNTARA-AYALA, 2002; MONTEIRO et al., 2012)

Tendo em vista a Política Nacional de Proteção e Defesa Civil - PNPDEC (2012) este trabalho tem por objetivo discutir como a mudança no paradigma de ações da Defesa Civil em trocar a gestão do desastre (resposta), pela gestão do risco (prevenção) impacta na resolução adotada pelo Brasil em tornar as cidades resilientes.

A análise a partir da revisão bibliográfica do conceito de resiliência urbana, de pesquisa documental sobre a Defesa Civil, a PNPDEC e o Programa 2040 - Gestão do Risco e Resposta a Desastres mostrou que nos anos de 2012 e 2013 houve uma série de repasses do governo federal para os estados para a execução de ações de prevenção.

\section{RESILIÊNCIA URBANA: AS RAÍZES DO CONCEITO}

O conceito de resiliência significa a "capacidade de um sistema sofrer uma perturbação e manter suas funções e controles" (GUNDERSON; HOLLING, 2001 apud JABAREEN, 2013). Segundo Janssen et al.(2006) o conceito foi introduzido por Holling (1973) na área da Ecologia para o estudo das dinâmicas dos ecossistemas.

Conforme Janssen et al. (2006) em meados da década de 80 o conceito passou a ser usado nas análises homem-meio ambiente; e posteriormente em outros campos, a exemplo da economia: resiliência econômica (ROGERS et al., 2012); da engenharia, "que engloba tanto a resistência e recuperação a partir de perturbações, embora a medição é focada exclusivamente na recuperação, quanto mais rápido a funcionalidade completa é restaurada, maior será a resiliência" (LIAO, 2012); da psicologia e psiquiatria, a capacidade do sujeito em lidar com as mudanças, os choques, as 
dificuldades e outros eventos ao decorrer de sua vida (RUTTER, 1987; KAPLAN, 1999 apud CHELLERI, 2012); e do social, que é entendida como a capacidade das comunidades humanas resistirem a choques externos resultado da variabilidade ambiental ou convulsões sociais, econômicas e políticas (ADGER, 2000 apud FOLKE, 2006).

A perspectiva homem-meio ambiente está ligada à Ecologia à medida que trata essa interação como um sistema sócio-ecológico - SES.

\begin{abstract}
"A teoria dos sistemas sócio-ecológicos explica como sistemas econômicos, sociais e ecológicos funcionam entre si. Sua intenção é entender a fonte e a função de mudanças sistêmicas, particularmente de mudanças transformativas em sistemas adaptáveis. Mudanças ecológicas, econômicas e sociais, que acontecem em diferentes velocidades e escalas espaciais, serão alvos de análises adaptativas" (GLASER et al. 2005 apud CABRAL, 2010).
\end{abstract}

Nos estudos urbanos, a resiliência na visão sócio-ecológica tem sido utilizada no debate do planejamento (LEICHENKO, 2011; PINHO, 2012; DESOUZA \& FLANERY, 2013; JABAREEN, 2013; MALALGODA, AMARATUNGA, HAIGH, 2013; LHOMME et al., 2013) especialmente voltado para as questões de desastres naturais, tem-se então a resiliência urbana, que é "a capacidade de uma cidade ou sistema urbano de absorver perturbações, mantendo a identidade, estrutura e processos-chave” (LEICHENKO, 2011).

Em outras palavras é dizer que uma cidade após sofrer algum desastre suas funções básicas funcionariam minimamente e que sua recuperação seria rápida e eficiente, além disso, a população saberia como agir diante da situação minimizando o número de vítimas. Ou seja, os efeitos negativos seriam reduzidos porque a cidade, conhecendo a quais fenômenos naturais está sujeita, estaria preparada e adaptada, tanto em termos materiais quanto humanos, dessa forma reencontraria um equilíbrio ou retornaria as condições iniciais de maneira eficaz.

Fontanals et al. (2012) enfatiza que deve-se considerar a resiliência como um processo transversal e não só como uma resposta imediata às adversidades, para o autor ser resiliente não significa ser invulnerável.

A Estratégia Internacional para Redução de Desastres das Nações Unidas - EIRD define uma cidade resiliente como "aquela que tem a capacidade de resistir, absorver e se recuperar de forma eficiente dos efeitos de um desastre e, de maneira organizada, prevenir que vidas e bens não sejam perdidos". (EIRD, 2010)

Ao considerar a realidade de países em desenvolvimento como o Brasil, a resiliência urbana pode induzir que retornar às condições anteriores é algo negativo dado as condições atuais, especialmente dos grupos sociais de baixa renda (MENDONÇA, 2011).

Todavia, o ambiente urbano pode tornar-se resiliente a partir do conhecimento das características sociais, físicas e econômicas do município e de uma ação efetiva do estado, sociedade civil e setor privado em prol da causa. Não se trata da criação de novas leis, mas da aplicação das já existentes para realocar pessoas que moram em áreas 
de risco, realizar obras estruturais, estimular uma cultura de prevenção a desastres naturais, etc.

\title{
3 A DEFESA CIVIL NO BRASIL
}

No Brasil, a Defesa Civil é a instituição responsável pelo planejamento e gestão de riscos e desastres naturais e incidentes tecnológicos. O Sistema de Defesa Civil foi criado no Brasil em 1942 no contexto da Segunda Guerra Mundial, mas apenas em 1988 foi organizado o Sistema Nacional de Defesa Civil - SINDEC, hoje parte do Ministério da Integração Nacional.

Os objetivos da Defesa Civil são

\begin{abstract}
"planejar e promover a defesa permanente contra as calamidades, integrando a atuação dos órgãos e entidades de planejamento, coordenação e execução das medidas de assistência às populações atingidas por fatores anormais adversos, assim como de prevenção ou recuperação de danos em Situação de Emergência ou em Estado de Calamidade Pública" (FERNANDES, 2012).
\end{abstract}

Na prática, entretanto, as ações da Defesa Civil, segundo Valencio (2010), "voltaram-se predominantemente para lidar com procedimentos padronizados na fase de resposta, relacionados à coordenação do cenário e atendimento a requerimentos burocráticos de avaliação de danos".

Em 2012, foi sancionada a lei 12.608 que institui a Política Nacional de Proteção e Defesa Civil - PNPDEC, que deve ser integrada às:

"políticas de ordenamento territorial, desenvolvimento urbano, saúde, meio ambiente, mudanças climáticas, gestão de recursos hídricos, geologia, infraestrutura, educação, ciência e tecnologia e às demais políticas setoriais, [...]" (BRASIL, 2012).

Uma das diretrizes da lei é o estímulo ao desenvolvimento de cidades resilientes, o que aponta para uma visão do desenvolvimento urbano mais focado nas ações de prevenção a desastres naturais do que nas de resposta.

\section{MEDIDAS PREVENTIVAS COMO CAMINHO PARA A RESILIÊNCIA}

Em termos de medidas preventivas o PNPDEC garante a profissionalização do agente de Defesa Civil através de cursos à distância e presenciais, a elaboração do Plano de Contingência de Proteção e Defesa Civil em nível municipal, dos Planos de Proteção e Defesa Civil nos três níveis de governo, o mapeamento de áreas de risco geotécnico, o monitoramento meteorológico e a consequente produção de alertas, o Sistema Integrado de Informações sobre Desastres - S2ID, dentre outros. 
No que se refere a orçamento, em 2012, o Ministério de Planejamento apresentou o Plano Nacional de Gestão de Riscos e Resposta a Desastres Naturais, que entre os anos de 2012 a 2014 possui $\mathrm{R} \$ 18,8$ bilhões em investimentos distribuídos da seguinte forma:

- $\mathrm{R} \$ 162$ milhões para o mapeamento;

- $\mathrm{R} \$ 362$ milhões para monitoramento e alerta;

- $\mathrm{R} \$ 2,6$ bilhões para resposta e

- $\mathrm{R} \$ 15,6$ bilhões para prevenção.

Ainda dentro deste escopo há o Programa 2040 - Gestão de Riscos e Respostas a Desastres que transfere recursos do governo federal para Estados e Municípios.

No ano de 2012, o Programa 2040 transferiu mais de R $\$ 360$ milhões para os estados investirem em ações de prevenção nas áreas de saneamento, urbanismo, ciência e tecnologia e segurança pública (Tabela 1).

Tabela 1 - Relação das ações preventivas que receberam recurso do Programa 2040 no ano de 2012.

\begin{tabular}{|c|c|c|c|}
\hline Ação & $\begin{array}{l}\text { Estados } \\
\text { Atendidos }\end{array}$ & Função & $\begin{array}{c}\text { Total } \\
\text { destinado no } \\
\text { ano }(\mathbf{R} \$)\end{array}$ \\
\hline $\begin{array}{l}\text { Apoio a Sistemas de Drenagem Urbana } \\
\text { Sustentável e de Manejo de Águas Pluviais } \\
\text { em Municípios com População Superior a } 50 \\
\text { mil Habitantes ou Integrantes de Regiões } \\
\text { Metropolitanas ou de Regiões Integradas de } \\
\text { Desenvolvimento Econômico }\end{array}$ & $\begin{array}{c}\text { Bahia, Goiás, } \\
\text { Maranhão, Mato } \\
\text { Grosso do Sul, } \\
\text { Minas Gerais, } \\
\text { Paraíba, Paraná, } \\
\text { Piauí, Rio de } \\
\text { Janeiro, Rio Grande } \\
\text { do Sul, Rondônia, } \\
\text { Santa Catarina, São } \\
\text { Paulo, Sergipe. }\end{array}$ & Saneamento & $275.323 .815,67$ \\
\hline $\begin{array}{l}\text { Construção da Barragem Serro Azul no } \\
\text { Estado de Pernambuco }\end{array}$ & Pernambuco & $\begin{array}{l}\text { Segurança } \\
\text { Pública }\end{array}$ & $77.539 .886,97$ \\
\hline $\begin{array}{l}\text { Apoio ao Planejamento e Execução de Obras } \\
\text { de Contenção de Encostas em Áreas Urbanas }\end{array}$ & $\begin{array}{l}\text { Amazonas, } \\
\text { Pernambuco, Rio de } \\
\text { Janeiro, São Paulo. }\end{array}$ & Urbanismo & $9.973 .214,35$ \\
\hline $\begin{array}{l}\text { Monitoramento e Alerta de Desastres } \\
\text { Naturais }\end{array}$ & $\begin{array}{l}\text { Distrito } \\
\text { Federal }\end{array}$ & $\begin{array}{l}\text { Ciência e } \\
\text { Tecnologia }\end{array}$ & 98.200 \\
\hline \multicolumn{3}{|l|}{ TOTAL } & $362.935 .116,99$ \\
\hline
\end{tabular}

Fonte: PORTAL DA TRANSPARÊNCIA GOVERNO FEDERAL (2014)

Observa-se a maior parte dos investimentos em obras de drenagem urbana e manejo de águas pluviais e da construção da barragem que colaboram para evitar ou atenuar o efeito negativo das enchentes. Já as obras de contenção de encostas evitam escorregamentos de terra e consequentemente previne danos humanos e materiais. $\mathrm{O}$ monitoramento e alerta de desastres naturais são fundamentais para o planejamento e para tomadas rápidas de decisão.

No ano de 2013, foram mais de R $\$ 740$ milhões transferidos destinados a investimentos nas áreas de urbanismo, segurança pública, gestão ambiental, saneamento e ciência e tecnologia (Tabela 2). 
Tabela 2 - Relação das ações preventivas que receberam recurso do Programa 2040 no ano de 2013

\begin{tabular}{|c|c|c|c|}
\hline Ação & $\begin{array}{c}\text { Estados } \\
\text { Atendidos }\end{array}$ & Função & $\begin{array}{l}\text { Total } \\
\text { destinado no } \\
\text { ano }(\mathbf{R} \$)\end{array}$ \\
\hline $\begin{array}{l}\text { Apoio a Sistemas de Drenagem Urbana } \\
\text { Sustentável e de Manejo de Águas Pluviais } \\
\text { em Municípios com População Superior a } 50 \\
\text { mil Habitantes ou Integrantes de Regiões } \\
\text { Metropolitanas ou de Regiões Integradas de } \\
\text { Desenvolvimento Econômico }\end{array}$ & $\begin{array}{c}\text { Bahia, Espírito } \\
\text { Santo, Goiás, Mato } \\
\text { Grosso do Sul, } \\
\text { Minas Gerais, } \\
\text { Paraíba, Paraná, } \\
\text { Pernambuco, Piauí, } \\
\text { Rio de Janeiro, Rio } \\
\text { Grande do Norte, } \\
\text { Rio Grande do Sul, } \\
\text { Rondônia, Santa } \\
\text { Catarina, São Paulo. }\end{array}$ & Saneamento & $468.443 .970,78$ \\
\hline $\begin{array}{l}\text { Apoio ao Planejamento e Execução de Obras } \\
\text { de Contenção de Encostas em Áreas Urbanas } \\
\text { (Prevenção de Riscos) }\end{array}$ & $\begin{array}{l}\text { Amazonas, Minas } \\
\text { Gerais, } \\
\text { Pernambuco, Rio de } \\
\text { Janeiro, Santa } \\
\text { Catarina, São Paulo. }\end{array}$ & Urbanismo & $80.909 .106,64$ \\
\hline $\begin{array}{l}\text { Construção da Barragem Serro Azul no } \\
\text { Estado de Pernambuco }\end{array}$ & Pernambuco & $\begin{array}{c}\text { Gestão } \\
\text { Ambiental }\end{array}$ & $70.000 .000,00$ \\
\hline $\begin{array}{c}\text { Obras de Macrodrenagem e Controle de } \\
\text { Erosão Marinha e Fluvial }\end{array}$ & $\begin{array}{l}\text { Amazonas, } \\
\text { Pernambuco, Santa } \\
\text { Catarina. }\end{array}$ & Urbanismo & $35.998 .772,27$ \\
\hline $\begin{array}{c}\text { Realização de Projetos e Obras para } \\
\text { Contenção ou Amortecimento de Cheias e } \\
\text { Inundações e para Contenção de Erosões } \\
\text { Marinhas e Fluviais }\end{array}$ & $\begin{array}{l}\text { Maranhão, } \\
\text { Pernambuco, Santa } \\
\text { Catarina. }\end{array}$ & $\begin{array}{l}\text { Gestão } \\
\text { Ambiental }\end{array}$ & $35.317 .850,64$ \\
\hline Apoio a Obras Preventivas de Desastres & $\begin{array}{l}\text { Ceará, Paraíba, } \\
\text { Pernambuco, Piauí, } \\
\text { Rio Grande do } \\
\text { Norte, Sergipe. }\end{array}$ & $\begin{array}{l}\text { Segurança } \\
\text { Pública }\end{array}$ & $35.145 .506,90$ \\
\hline $\begin{array}{c}\text { Construção da Barragem Serro Azul no } \\
\text { Estado de Pernambuco }\end{array}$ & Pernambuco & $\begin{array}{l}\text { Segurança } \\
\text { Pública }\end{array}$ & $12.460 .113,03$ \\
\hline $\begin{array}{c}\text { Monitoramento e Alerta de Desastres } \\
\text { Naturais CEMADEN }\end{array}$ & $\begin{array}{l}\text { Alagoas, Distrito } \\
\text { Federal. }\end{array}$ & $\begin{array}{l}\text { Ciência e } \\
\text { Tecnologia }\end{array}$ & $3.270 .000,00$ \\
\hline $\begin{array}{l}\text { Implantação do Centro Nacional de } \\
\text { Monitoramento e Alerta de Desastres } \\
\text { Naturais - CEMADEN }\end{array}$ & $\begin{array}{l}\text { Distrito } \\
\text { Federal }\end{array}$ & $\begin{array}{l}\text { Ciência e } \\
\text { Tecnologia }\end{array}$ & $1.130 .000,00$ \\
\hline $\begin{array}{c}\text { Obras para Controle de Cheias de Erosões } \\
\text { Marinhas e Fluviais }\end{array}$ & Paraná & $\begin{array}{c}\text { Gestão } \\
\text { Ambiental }\end{array}$ & $300.000,00$ \\
\hline \multicolumn{3}{|l|}{ TOTAL } & $742.975 .320,26$ \\
\hline
\end{tabular}

Fonte: PORTAL DA TRANSPARÊNCIA GOVERNO FEDERAL (2014)

Em comparação com o ano de 2012, nota-se claramente que no ano de 2013 houve mais ações que receberam recursos. Foram obras relacionadas à drenagem urbana, macrodrenagem, barragem, prevenção de desastres, contenção de encostas em áreas urbanas, bem como contenção e controle de erosão marinha e fluvial, de cheias e inundações. Houve significativo incremento no valor de recursos destinados ao monitoramento e alerta, importantes para o planejamento como dito anteriormente, mas, 
sobretudo para salvar vidas desde que a população seja ensinada como proceder e para onde ir quando alertada.

Nota-se na política orçamentária o grande investimento destinado à prevenção, outro elemento que reforça a mudança de paradigma. Não trata de desmerecer a gestão de desastres, ela é vital para a recuperação das cidades. Todavia, o foco em preparação visa à adoção de medidas de adaptação, redução de vulnerabilidades para que nos eventos de desastres naturais a fase de resposta ser mais rápida.

O caráter multidisciplinar da resiliência urbana entende a cidade como um sistema sócio-ecológico em que sistemas econômicos, sociais e ecológicos relacionam-se entre si, portanto é necessário que os investimentos atinjam outras áreas também.

A melhoria significativa nas cidades se dará quando a Defesa Civil participar das políticas públicas intersetoriais em conjunto com a habitação, saúde, meio ambiente, educação e cultura, assistência social, obras públicas, dentre outras. Ao participar da gestão territorial a população não será mais submetida a soluções paliativas e o ambiente urbano tornar-se-á cada vez mais resiliente.

É importante que o caráter multidisciplinar das políticas, que envolvem diversas secretarias, não seja motivo para a manutenção da situação de risco. Valencio (2010) diz que a divisão das funções públicas em setores foi pensada para ser benéfica, para trazer qualidade de vida ao cidadão, mas diante de tragédias como os desastres naturais essa setorização faz com que a culpa seja de todos e ao mesmo tempo de ninguém. Ou seja, exime a culpa do Estado.

\section{CONCLUSÃO}

Os investimentos em preparação e prevenção do Programa 2040 ilustra em termos práticos a mudança de paradigma da Defesa Civil. Trata-se também de uma questão de racionalização de gastos, pois, apoiar medidas preventivas é mais barato do que reconstruir grandes áreas devastadas.

De forma que é necessário que haja uma consolidação com novos investimentos em áreas integrantes da política de proteção e defesa civil como meio ambiente, educação e cultura, saúde pública, ordenamento territorial, etc.

Todas as ações que apoiam tomadas de decisão precisam de investimento constante a exemplo do mapeamento de áreas de risco geotécnico, o monitoramento meteorológico, a produção de alertas e o Sistema Integrado de Informações sobre Desastres.

A profissionalização do agente de Defesa Civil é de suma importância para capacitá-lo a integrar-se e articular-se com as demais secretarias, assim como prevê o PNPDEC, para realizar uma gestão territorial responsável que envolva além do setor público, o setor privado e a sociedade civil, de maneira a promover a resiliência nos municípios brasileiros.

\section{AGRADECIMENTO}

À CAPES, pelo apoio recebido.

À Fundação de Amparo à Pesquisa do Estado de São Paulo (FAPESP), processo ${ }^{\circ}$ 2014/16362-1, pelo apoio na divulgação da pesquisa. 


\section{REFERÊNCIAS}

ALCÁNTARA-AYALA, I. Geomorphology, natural hazards, vulnerability and prevention of natural disasters in developing countries. Geomorphology, Outubro. 2002. v. 47, n. 2-4, p. $107-124$.

BRASIL. Lei $\mathbf{n}^{\mathbf{0}}$ 12.608, de 10 de abril de 2012. Institui a Política Nacional de Proteção e Defesa Civil - PNPDEC.

- Portal da Transparência Governo Federal. Disponível em: <www.portaltransparencia.gov.br/>. Acesso em: 12 fev. 2014.

CABRAL, N. W. S. S. Zona costeira resiliente: um estudo sócio-ecológico no nordeste paraense. Belém: Universidade Federal do Pará, Núcleo de Altos Estudos Amazônicos Programa de Pós-Graduação em Desenvolvimento Sustentável do Trópico Úmido, 2010. Tese (Doutorado). Disponível em: <http://repositorio.ufpa.br/jspui/handle/2011/2722〉.

CEPED UFSC. Atlas brasileiro de desastres naturais 1991 a 2010: volume Brasil. Florianópolis: Universidade Federal de Santa Catarina. Centro Universitário de Estudos e Pesquisas sobre Desastres, 2012.

CHELLERI, L. From the «Resilient city» to urban resilience. a review essay on understanding and integrating the resilience perspective for urban systems. Documents d'Analisi Geografica, 2012. v. 58, n. 2, p. 287-306.

DESOUZA, K. C.; FLANERY, T. H. Designing, planning, and managing resilient cities: A conceptual framework. Cities, 2013. v. 35, p. 89-99.

EIRD - Estratégia Internacional para Redução de Desastres. Disponível em: < http://eird.org/curso-brasil/docs/modulo7/4.SEDEC-Cidades-Resilientes.pdf >. Acesso em: 03 abril 2013.

FERNANDES, S.F. Civil Defense and bad news. Mundo da Saude 36, no. 1 (2012): 71-74. ISSN 1980-3990 (versão eletrônica)

FIALHO, W. M. B. ; MOLION, L. C. B. . Eventos Extremos: Alagoas Junho de 2010. In: IV Encontro Sul-Brasileiro de Meteorologia - ESBM 2011, 2011, Pelotas - RS. Anais do IV Encontro Sul-Brasileiro de Meteorologia - ESBM 2011, 2011. Disponível em: < http://wp.ufpel.edu.br/meteoro/files/2011/05/wendell_barbosa_1.pdf > Acesso em: 10 jan. 2014.

FOLKE, C. Resilience: The emergence of a perspective for social-ecological systems analyses. Global Environmental Change, ago. 2006. v. 16, n. 3, p. 253-267. . Acesso em: 8 mar. 2013.

FONTANALS, L. et al., Mejorando la Resiliencia de las ciudades: Conocimiento industrial aplicado a la Gestión de la Ciudad. In: Congreso Nacional del Medio Ambiente (CONAMA), 2012, Madrid. Disponível em: < http://www.conama2012.conama.org/web/generico.php?idpaginas=\&lang=es\&menu=90\&refer er $=1 \& \mathrm{id}=285 \&$ tipo=C\&op=view\&from=view_personas $>$

HOLLING, C. S. Resilience and Stability of Ecological Systems. ArticleType: research-article / Full publication date: 1973 / Copyright (C) 1973 Annual Reviews: Annual Review of Ecology and Systematics, 1 jan. 1973. v. 4, p. 1-23. . Acesso em: 9 jan. 2014.

JABAREEN, Y. Planning the resilient city: Concepts and strategies for coping with climate change and environmental risk. Cities, abr. 2013. v. 31, p. 220-229. . Acesso em: 19 abr. 2013.

JANSSEN, M. A. et al. Scholarly networks on resilience, vulnerability and adaptation within the human dimensions of global environmental change. Global Environmental Change, Agosto. 2006. v. 16, n. 3, p. 240-252. . Acesso em: 8 out. 2014. 
LEICHENKO, R. Climate change and urban resilience. Current Opinion in Environmental Sustainability, 2011. v. 3, n. 3, p. 164-168.

LIAO, K.-H. A theory on urban resilience to floods-A basis for alternative planning practices. Ecology and Society, 2012. v. 17, n. 4.

MALALGODA, C.; AMARATUNGA, D.; HAIGH, R. Creating a disaster resilient built environment in urban cities: The role of local governments in Sri Lanka. International Journal of Disaster Resilience in the Built Environment, 22 fev. 2013. v. 4, n. 1, p. 72-94.

MENDONÇA, F. Riscos, vulnerabilidade e resiliencia sociambientais urbanas: inovações na análise geográfica. Revistada Associação Nacional de Pesquisa e Pós-Graduação em Geografia (ANPEGE), v.7, n. 1, número especial, p.111-118, Outubro 2011. DOI: 10.5418/RA2011.0701. 0010

MI - MINISTÉRIO DA INTEGRAÇÃO NACIONAL. Cidades Resilientes. Disponível em: < http://www.mi.gov.br/cidadesresilientes/ >. Acesso em: 07 dez. 2013.

Principais Ações do Programa 2040 - Gestão de Riscos e Resposta a Desastres. Disponível em: < http://www.integracao.gov.br/acoes-2040 >. Acesso em: 17 ago. 2014.

MONTEIRO, J. B.; PINHEIRO, D. R. C.. O desastre natural como fenômeno induzido pela sociedade: abordagens teóricas e metodologias operacionais para identificação/mitigação de desastres naturais. Revista de Geografia da IFJP, v. 2, p. 1-9, 2012. Disponível em: < http://www.ufjf.br/revistageografia/files/2012/10/O-DESASTRE-NATURAL-COMO-

FEN\%C3\%94MENO-INDUZIDO-PELA-SOCIEDADE-ABORDAGENS-TE\%C3\%93RICASE-METODOL\%C3\%93GICAS-OPERACIONAIS-PARA-

IDENTIFICA\%C3\%87\%C3\%83OMITIGA\%C3\%87\%C3\%83O-DE-DESASTRES-

NATURAIS.pdf >. Acesso em: 14 maio 2013.

MP-MINISTÉRIO DO PLANEJAMENTO. Apresentação do Plano Nacional de Gestão de Riscos e Resposta a Desastres Naturais - Brasília/DF. 08/08/2012. Disponível em: < http://www.planejamento.gov.br/secretarias/upload/Arquivos/pronunciamentos/apresentacaoes/ 2012/120808_Plano_Nac_Risco_2.pdf > Acesso em10/11/2013.

PINHO, P. A Cidade Resiliente. In: FIGUEIREDO, António Manuel; PENEBAD, Jose Manuel Peña; ÁLVAREZ, Enrique José Varela (Org.). Retos de la acción de gobierno para las ciudades del siglo XXI - Desafíos da governação das cidades do século XXI. Porto: Eixo Atlântico do Nordeste Peninsular, 2012. Cap. 5, p. 283 - 304. Disponível em: < http://eixoatlantico.com/eixo/sites/default/files/Retos\%20de\%20la\%20accion.pdf\#page=285 >. Acesso em: 03 jan. 2013.

ROGERS, C. D. F. et al. Resistance and resilience - paradigms for critical local infrastructure. Proceedings of the ICE - Municipal Engineer, 1 jun. 2012. v. 165, n. 2, p. $73-$ 83. Acesso em: 15 mar. 2013.

VALENCIO, N. Desastres, ordem social e planejamento em defesa civil: o contexto brasileiro. Saude soc., São Paulo, v. 19, n. 4, Dec. 2010. Disponível em: $<$ http://www.scielo.br/scielo.php?script=sci_arttext\&pid=S010412902010000400003\&lng=en\&nrm=iso>. Accesso em: 08 dez. 2014. 